\title{
Knowledge, attitude and practice of post-natal exercises among post- natal women from a tertiary care centre, South India
}

\author{
Raja Sundaramurthy $^{1}$, Sasikala Kathiresan ${ }^{2 *}$, Sriandaal Venkateshvaran $^{3}, \operatorname{Surya}_{K^{\prime}} \operatorname{Kannan}^{2}$
}

\author{
${ }^{1}$ Department of Microbiology, Velammal Medical College Hospital and Research Institute, Anuppanadi, Madurai, \\ Tamil Nadu, India \\ ${ }^{2}$ Department of Obstetrics and Gynecology, Velammal Medical College Hospital and Research Institute, Anuppanadi, \\ Madurai, Tamil Nadu, India \\ ${ }^{3}$ Department of Preventive and Social Medicine, Velammal Medical College Hospital and Research Institute, \\ Anuppanadi, Madurai, Tamil Nadu, India
}

Received: 19 February 2020

Accepted: 28 February 2020

*Correspondence:

Dr. Sasikala Kathiresan,

E-mail: sasisganesh@gmail.com

Copyright: (C) the author(s), publisher and licensee Medip Academy. This is an open-access article distributed under the terms of the Creative Commons Attribution Non-Commercial License, which permits unrestricted non-commercial use, distribution, and reproduction in any medium, provided the original work is properly cited.

\begin{abstract}
Background: Stressful puerperal period and its complications can be prevented by adequate care, proper diet and exercises. Though studies have indicated post-natal exercises (PNE) is helpful in reducing the effect of perineal muscle weakness, many women are unaware of its full benefits. This study is planned to know the knowledge, attitude and practice of PNE in post-natal mothers.

Methods: Descriptive cross-sectional study was conducted in department of obstetrics and gynecology for 3 months (November 2019 to January 2020). 160 post-natal women were enrolled with convenient sampling. Structured questionnaire was used to collect socio-demographic and obstetric details, source of information, knowledge, attitude and practice of PNE.

Results: A total $58.8 \%$ of this study participants revealed health care professions were the commonest source of information. More than half of them had adequate knowledge but still many were not aware of full benefits of PNE as only $3-5 \%$ aware of other benefits like prevention of urinary incontinence. $98.8 \%$ of the women's felt PNE is essential and $62.5 \%$ felt household work hinder them doing. $92.5 \%$ said they will emphasize the importance of PNE to others. No significant correlation found between socio-demographic characters and knowledge, attitude and practice of PNE. Conclusions: Though most of the study population had adequate knowledge and positive attitude, household activities were the commonest barrier in doing PNE. Educating and creating awareness among family members is essential to improve the adherence. Continuing influence by health care workers during antenatal and postnatal period will be the key for improving adherence.
\end{abstract}

Keywords: Knowledge attitude and practice, Postnatal exercise, Postnatal women

\section{INTRODUCTION}

First six weeks following childbirth known as puerperal period is a stressful for any women due to physiological and psychological adaptations which happens during this period and pelvic organ returns to pre pregnant state. ${ }^{1}$ Inadequate care during this period will lead to most of the complications like perineal muscle weakness leading to bladder incontinence, back pain, postpartum psychosis etc. $^{2-5}$ These complications can be prevented by adequate care like proper diet and exercises. ${ }^{5-7}$

Post-natal exercises (PNE) like Kegel's, abdominal and breathing exercises helps to improve the pelvic floor and 
abdominal muscle tone, bowel and bladder function and cardio vascular fitness. ${ }^{5,7,8}$ Also PNE will have the positive influence on preventing depression and other psychological disorders as it keeps the mother more relaxed. $^{2}$

PNE can be started soon after birth with simple exercises and gradually progress to advance depending on the level of comfort. ${ }^{9,10}$ Though many studies have indicated that PNE plays the major role in preventing complications like bladder incontinence, many women are unaware of full benefits of postnatal exercise and also some of the customs and superstitions existing in the community will be prohibiting the postnatal women from performing exercises. $^{5,6,8}$

This study is planned to know the knowledge, attitude and practice of the post-natal mothers related to PNE to make a positive culture of adherence to PNE among all of the postnatal mothers.

\section{METHODS}

This descriptive cross-sectional study was carried out in the department obstetrics and gynecology of the tertiary care centre, South India, spanned over a period of 3 months (November 2019 to January 2020) after attaining the Institutional Ethics Committee approval. After getting the written informed consent 160 postnatal women who have attended the obstetrics and gynecology and pediatric outpatient department (OPD) were enrolled in the study with convenient sampling. Patient unaware of post-natal exercise, 1 st post-natal period and women not willing to participate were excluded from the study

Structured proforma was used to collect socio demographic details like religion, education, occupation, income, type of family and domicile nature of the study participants. Followed by obstetric history like parity, mode of delivery and children were collected.

Source of information for PNE was collected as next part. Knowledge of the participants about PNE were collected with the structured questionnaire which includes when to start, how frequently needed, types of PNE, benefits and disadvantage of PNE. Attitude and practice of the study population also collected with the structured questionnaire which includes which includes barriers preventing PNE, way to improve adherence, health professions and emphasis of PNE during antenatal postnatal visit.

\section{Statistical analysis}

Statistical analysis was carried out using SPSS version 20. Descriptive data were expressed as number and percentage. Chi square test was used to analysis the correlation of socio-economic characters with knowledge, attitude and practice of the study population. Each question in the knowledge, attitude and practical was given 1 mark. Total score of 5 was there in each category, mothers those who scored $>3$ marks were considered as good.

\section{RESULTS}

A total of 160 women who have attended OBG and Paediatric OPD department of the tertiary care centre were enrolled in the study. Age distribution of the study population was 22-38 years with the mean age of $28.2 \pm 4.1$ years.

\section{Table 1: Socio-demographic characters of the} study population.

\begin{tabular}{|c|c|c|}
\hline Socio- demographic status & Number & $\%$ \\
\hline \multicolumn{3}{|l|}{ Religion } \\
\hline Hindu & 132 & $82.5 \%$ \\
\hline Christian & 4 & $2.5 \%$ \\
\hline Muslim & 24 & $15 \%$ \\
\hline \multicolumn{3}{|l|}{ Education } \\
\hline Illiterate & 2 & $1.2 \%$ \\
\hline Primary & 2 & $1.2 \%$ \\
\hline Middle & 14 & $8.8 \%$ \\
\hline High school & 60 & $37.5 \%$ \\
\hline Graduation and above & 82 & $51.3 \%$ \\
\hline \multicolumn{3}{|l|}{ Occupation } \\
\hline Unemployed & 72 & $45 \%$ \\
\hline Govt. service & 4 & $2.5 \%$ \\
\hline Self & 14 & $8.8 \%$ \\
\hline Private employed & 70 & $43.7 \%$ \\
\hline \multicolumn{3}{|l|}{ Income in rupees } \\
\hline Not applicable & 72 & $45 \%$ \\
\hline$<2500$ & - & - \\
\hline $2501-5000$ & - & - \\
\hline $5001-10000$ & 42 & $26.3 \%$ \\
\hline Above 10000 & 46 & $28.7 \%$ \\
\hline \multicolumn{3}{|l|}{ Type of family } \\
\hline Nuclear & 134 & $83.8 \%$ \\
\hline Joint & 26 & $16.2 \%$ \\
\hline \multicolumn{3}{|l|}{ Domicile } \\
\hline Rural & - & - \\
\hline Semi urban & 84 & $52.5 \%$ \\
\hline Urban & 76 & $47.5 \%$ \\
\hline
\end{tabular}

Majority of the participants were belonging to Hindu religion $(82.5 \%)$. Half of the study population were graduates $(51.3 \%)$. Unemployed and private employed were distributed equally $(45,43.7 \%$ respectively). $55 \%$ of the participants had income above Rs. 5000. 83.8\% belongs to nuclear family. All of them were either from semi urban and/ or urban area. Table 1 represents the socio demographic status of this study population.

Obstetric history of study participants showed $51.2 \%$ had caesarean; $48.8 \%$ had normal vaginal delivery. $66.3 \%$ of participants had two children and $33.7 \%$ had one child. 
A total $58.8 \%$ of the study participants were given that health care professions as a source of information for PNE followed by TV (12.5\%), social media (11.2\%) and friends $(11.2 \%)$. Figure 1 represents the source of information regarding postnatal exercise among this study population.

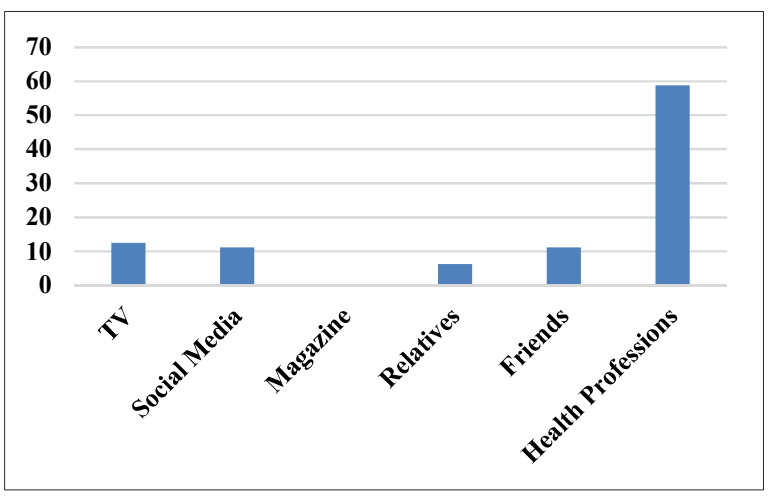

Figure 1: Source of information regarding post-natal exercise.

Table 2: Knowledge about postnatal exercise among the study populations.

\begin{tabular}{|c|c|c|}
\hline Knowledge & Number & $\%$ \\
\hline \multicolumn{3}{|l|}{ When to start PNE? } \\
\hline Immediately & 88 & $55 \%$ \\
\hline After 2 weeks & 64 & $40 \%$ \\
\hline After 1 month & 6 & $3.8 \%$ \\
\hline After 6 months & 2 & $1.2 \%$ \\
\hline \multicolumn{3}{|c|}{ How frequently PNE is needed? } \\
\hline Once a day & 106 & $66.3 \%$ \\
\hline Once a week & 30 & $18.7 \%$ \\
\hline 4-5 days/weeks & 24 & $15 \%$ \\
\hline Once a month & - & - \\
\hline \multicolumn{3}{|c|}{ What are the types of PNE you know? } \\
\hline Breathing exercise & 124 & $77.5 \%$ \\
\hline Kegel's exercise & 30 & $18.6 \%$ \\
\hline Pelvic floor exercise & 46 & $28.7 \%$ \\
\hline Abdominal exercise & 88 & $55 \%$ \\
\hline \multicolumn{3}{|c|}{ Benefits of PNE in preventing } \\
\hline Uterine prolapse & 156 & $97.5 \%$ \\
\hline Diastasis recti & 8 & $5 \%$ \\
\hline Bowel incontinence & 8 & $5 \%$ \\
\hline Urinary incontinence & 6 & $3.7 \%$ \\
\hline \multicolumn{3}{|c|}{ Disadvantage of not performing PNE? } \\
\hline Weight gain & 138 & $86.3 \%$ \\
\hline High BP & - & - \\
\hline Back pain & 34 & $21.2 \%$ \\
\hline Postpartum depression & 20 & $12.5 \%$ \\
\hline
\end{tabular}

Among the study populations $55 \%$ of women were aware of when to start PNE; 66.3\% was aware of how frequently PNE needed; breathing exercise $(77.5 \%)$ followed by abdominal exercise $(55 \%)$ were the well- known types of exercise among the $=$ is study populations. $97.5 \%$ known that prevention of uterine prolapse was one of the benefits of PNE. $86.3 \%$ women told that weight gain was the most common disadvantage of not performing PNE. Table 2 represents knowledge about PNE among this study populations.

Table 3: Attitude about postnatal exercise among this study populations.

\begin{tabular}{|c|c|c|}
\hline Attitude & Number & $\%$ \\
\hline \multicolumn{3}{|l|}{ PNE is necessary } \\
\hline Agree & 158 & $98.8 \%$ \\
\hline Disagree & 2 & $1.2 \%$ \\
\hline \multicolumn{3}{|c|}{ I adhere to PNE regularly } \\
\hline Agree & 78 & $48.7 \%$ \\
\hline Disagree & 82 & $51.3 \%$ \\
\hline \multicolumn{3}{|c|}{ I feel guilty on omitting PNE } \\
\hline Agree & 98 & $61.3 \%$ \\
\hline Disagree & 62 & $38.7 \%$ \\
\hline \multicolumn{3}{|c|}{ Health professions having positive influence } \\
\hline Agree & 152 & $95 \%$ \\
\hline Disagree & 8 & $5 \%$ \\
\hline \multicolumn{3}{|c|}{ What prevents you to do PNE? } \\
\hline Pain & 14 & $8.8 \%$ \\
\hline Fear of injury & 22 & $13.7 \%$ \\
\hline Baby care & 32 & $20 \%$ \\
\hline Household work & 100 & $62.5 \%$ \\
\hline Fatigue & 14 & $8.7 \%$ \\
\hline Lack of information & 8 & $5 \%$ \\
\hline \multicolumn{3}{|c|}{ How adherence to PNE can be improved? } \\
\hline Motivation & 40 & $25 \%$ \\
\hline Training and education & 20 & $12.5 \%$ \\
\hline Display boards & 4 & $2.5 \%$ \\
\hline $\begin{array}{l}\text { Awareness during } \\
\text { antenatal visit }\end{array}$ & 106 & $66.2 \%$ \\
\hline
\end{tabular}

Table 4: Practice about postnatal exercise among this study populations.

\begin{tabular}{|c|c|c|}
\hline Practice & Number & $\%$ \\
\hline \multicolumn{3}{|c|}{ How frequency of you are doing PNE } \\
\hline Daily & 90 & $56.3 \%$ \\
\hline Once a week & 58 & $36.3 \%$ \\
\hline Occasionally & 12 & $7.5 \%$ \\
\hline \multicolumn{3}{|c|}{ Have you experienced benefits of PNE } \\
\hline Yes & 142 & $88.8 \%$ \\
\hline No & 18 & $11.2 \%$ \\
\hline \multicolumn{3}{|c|}{ Antenatal visits having positive influence on PNE } \\
\hline Yes & 98 & $61.3 \%$ \\
\hline No & 62 & $38.7 \%$ \\
\hline \multicolumn{3}{|c|}{ Postnatal visits having positive influence on PNE } \\
\hline Yes & 136 & $85 \%$ \\
\hline No & 24 & $15 \%$ \\
\hline \multicolumn{3}{|c|}{ I will emphasize PNE to others } \\
\hline Yes & 148 & $92.5 \%$ \\
\hline No & 12 & $7.5 \%$ \\
\hline
\end{tabular}


A total $98.8 \%$ of the women's felt PNE is essential but only $48.7 \%$ agreed for adherence to PNE. $61.3 \%$ said that they feel guilty when omitting PNE. 95\% had the opinion that health professions had the positive influence. Analysis of reason which prevents the PNE $62.5 \%$ felt that household work followed by baby care $(20 \%)$ and fear of injury (13.7\%). The way to improve adherence $66.2 \%$ felt that emphasis during the antenatal visit was important followed by motivation $(25 \%)$. Table 3 represents attitude about PNE among this study populations.

Table 5: Cross tabulation between socio demographic characteristic and total score of knowledge and practice.

\begin{tabular}{|l|llll|}
\hline \multirow{2}{*}{ Socio demographic data } & Total knowledge & & Total practice & Sig. (2 tailed) \\
\hline Age & Test statistic & Sig. (2 tailed) & Test statistic & Sich \\
\hline Religion & 0.731 & 0.866 & 0.566 & 0.904 \\
\hline Education & 0.380 & 0.827 & 1.131 & 0.568 \\
\hline Occupation & 3.544 & 0.471 & 1.045 & 0.903 \\
\hline Income & 6.026 & 0.110 & 0.952 & 0.813 \\
\hline Family type & 3.550 & 0.169 & 0.68 & 0.967 \\
\hline Domicile & 0.373 & 0.402 & 1.035 & 0.309 \\
\hline Parity & 0.664 & 0.415 & 0.334 & 0.563 \\
\hline Mode of delivery & 0.396 & 0.529 & 0.451 & 0.502 \\
\hline
\end{tabular}

A total $56 \%$ of the women practising PNE daily and $36.3 \%$ practicing once a week; $88.8 \%$ experienced the benefits; $62 \%$ and $85 \%$ have felt that antenatal visit and postnatal visit respectively had the positive influence. 92.5\% said that they will emphasise the importance of PNE to others. Table 4 represents practice about PNE among this study populations.

Correlation of socio demographic character with knowledge and practice showed no significant association $(\mathrm{p}>0.05)$. Table 5 represents cross tabulation between socio demographic characteristic and total score of knowledge and practice.

\section{DISCUSSION}

Total of 160 women with the mean age of $28.2 \pm 4.1$ years were enrolled in this study, of which $66.3 \%$ were multigravida, $51.2 \%$ had caesarean mode of delivery. This study population group was discordant with the study group of Jawaher et al, Mbada et al, most of their study population were vaginal delivery. ${ }^{6,8}$ As caesarean delivery was predominate in this set up, we need to create more awareness among this study population to change the attitude from conservative cultural perceptions and myths that exercise is unsafe for pregnant women which makes them afraid to participate in view of fear that it will have negative impact on their health.

On analyzing the source of information for PNE, 58.8\% of this study participants were revealed that health care professions were the most common mode followed by TV and social media (23.7\%). This study findings were in discordant with many other study results like Jawaher et al, Ibrahim et al, Alharqi et al, which media was the most common source reported for information of PNE. ${ }^{6,11,12}$ This study findings may be attributed to the protocol of the tertiary care centre which is having the practice of getting physiotherapy consultant for all postnatal mother delivered in the hospital and also the consultants and nursing staffs will emphasize the importance of PNE during antenatal and postnatal visit.

On analyzing the knowledge about PNE, 55\% of this study population were aware of when to start PNE and $66.3 \%$ aware of how frequently it needed. This findings were in concordance with Alharqi et al (65\% awareness) but discordant with Sreenivasan A et al, Ashok VG et al, $\left(6.7 \%\right.$ and $22 \%$ awareness respectively). ${ }^{4,12,13}$ Better knowledge in this study may be attributed to the domicile nature of this study population as all of them either from semi urban or urban area and also more than half of this study population were graduates.

Breathing exercises (77.5\%) followed by abdominal exercises $(55 \%)$ were the well-known types of exercises among this study populations. $81.4 \%$ and $71.3 \%$ not aware of Kegel's and pelvic floor exercises respectively. This results were in concordance with Wojno et al. ${ }^{14}$ There was a knowledge gap about other exercises, it may be due to, still people may have belief in the community that pelvic floor exercises should not be carried out after caesarean section, which was the major group who may not interested in knowing other exercises.

A total $97.5 \%$ known that prevention of uterine prolapse was one of the benefits of PNE. In contrast only 3-5\% aware of other benefits like prevention of bowel, urinary incontinence. On analyzing disadvantage of not performing PNE, weight gain $(86.3 \%)$ was the most common followed by back pain $(21.2 \%)$. Here also there was a knowledge gap found among this study populations related to advantage and disadvantage which may be rectified by proper training and education. 
On analysis the attitude of this study population about PNE revealed that $98.8 \%$ of the women's felt PNE is essential and $61.3 \%$ felt that they feel guilt when omitting PNE. This positive attitude may be attributed to the health professions who constantly encouraging and emphasizing the importance of PNE among post-natal mothers which was also indicated by this study group as $95 \%$ had the opinion that health professions had the positive influence.

Analysis of barrier which prevents the PNE revealed that $62.5 \%$ felt that household work followed by baby care $(20 \%)$ and fear of injury (13.7\%). This was in discordant with study result of Alharqi et al, who reported that lack of information and fear of injury were the most common barrier. ${ }^{12}$ This study finding may be attributed due to the nuclear family nature of this study population. So, help from others (friend/family) may have the positive impact in this study setup. Analyzing the way to improve adherence $66.2 \%$ felt that emphasis during the antenatal visit was important followed by motivation $(25 \%)$. This study finding emphasis that gaining the confidence of the mother related to false beliefs regarding PNE is very important during both antenatal and postnatal visit to improve the attitude.

On analyzing the practice of PNE among this study population revealed that $56 \%$ practicing PNE daily and $36.3 \%$ practicing once a week; $88.8 \%$ experienced the benefits; $62 \%$ and $85 \%$ have felt that antenatal visit and postnatal visit respectively had the positive influence. $92.5 \%$ said that the will emphasize the importance of PNE to others. This may be attributed to the antenatal and postnatal health education provided to the women through the health care professions.

In contrast to the other studies Jawaher et al, Alharqi et al, who have reported positive association between women's education and knowledge about PNE, this studies report suggest that there was no significant correlation between any socio demographic character with knowledge, attitude and practice of PNE. ${ }^{6,12}$ This may be due to nature of this study population which had more than $50 \%$ educated and all of them either from urban or semi urban area and protocol of the health system had the positive influence on adherence to PNE which was shown by the positive attitude almost by all patients.

\section{CONCLUSION}

More than half of this study population had adequate knowledge about PNE but still many of them were not aware of the full benefits of PNE. As most of them said health care professions were the main source of information, it is the prime role to throw light on the importance of postnatal exercise and emphasis to adherence to PNE among all the post-natal mothers. Most of study population had the positive attitude but felt that household activities were the most common barrier.
Education and creating awareness among family members and friends of the post-natal mothers are also very important to improve the adherence. More than half were also practising the exercises and said that they will emphasise the importance of PNE to others. This culture making will have a positive impact among the community in relation to women health. No association was found among socio-demographic character with knowledge, attitude and practice. So continuing influence by health care workers will be the key for improving the adherence rate among this study populations.

\section{ACKNOWLEDGMENTS}

Authors would like to thank Dr Saritha, CRRI for her valuable contribution in collection of data.

Funding: No funding sources Conflict of interest: None declared

Ethical approval: The study was approved by the Institutional Ethics Committee (Ref No.: VMCIEC/ 215/ 2019)

\section{REFERENCES}

1. Evenson KR. Towards an understanding of change in physical activity from pregnancy through postpartum. Psychol Sport Exerc. 2011;12(1):36-45.

2. Kołomańska-Bogucka D, Mazur-Bialy AI. Physical activity and the occurrence of postnatal depression-a systematic review. Medicina (Mex). 2019;55(9):560.

3. Hammer RL, Perkins J, Parr R. Exercise during the childbearing year. J Perinat Educ. 2000;9(1):1-14.

4. Khaskheli MN, Baloch S, Sheeba A. Risk factors and complications of puerperal sepsis at a tertiary healthcare centre. Pak J Med Sci. 2013;29(4):972-6.

5. Sreenivasan A. A study to assess the knowledge, practice and attitude of postnatal mother regarding postnatal exercise in government headquarters hospital, Kumbakonam. Int $\mathrm{J}$ Adv Sci Res. 2017;2(4):32-5.

6. Alharbi JH. Knowledge, attitude and practices of kegel exercise among postnatal women, in $\mathrm{Al}$ Madinah Al Munawarah, Saudi Arabia. Int J Nurs Didact. 2019;09(06):01-10.

7. Sahu S, Dash M. Knowledge and attitude of postnatal mothers regarding postnatal exercise. J Midwifery, Women Health Gynaecol Nurs. 2019;2(1):1-5.

8. Mbada CE, Adebayo OE, Awotidebe TO, Faremi FA, Oginni MO, Ogundele AO, et al. Practice and pattern of antenatal and postnatal exercise among nigerian women: a cross-sectional study. Int $\mathbf{J}$ Womens Health Reprod Sci. 2015;3(2):93-8.

9. Daley AJ, Jolly K, Sharp DJ, Turner KM, Blamey $\mathrm{RV}$, Coleman S, et al. The effectiveness of exercise as a treatment for postnatal depression: study protocol. BMC Preg Childbirth. 2012;12:45.

10. Adeniyi AF, Ogwumike OO, Bamikefa TR. Postpartum exercise among nigerian women: issues 
relating to exercise performance and self-efficacy. ISRN Obstet Gynecol. 2013:Article ID 294518,

11. Ibrahim WA. Assess levels of knowledge, attitude and practice of the married women about pelvic floor muscles exercise. Inter J Sci Res. 2015;6(10):5.

12. Alharqi HM, Albattawi JA. Assessment of knowledge and attitude of women towards postpartum exercise. IOSR J Nurs Health Sci. 2018;7(1):16-20.

13. Mohamed A, Ashok V. Knowledge, practice and attitude of postnatal mothers towards postnatal exercises in a rural area of Tamil Nadu. Indian $\mathbf{J}$ Forensic Community Med. 2019;6(3):134-7.
14. Wojno A, Terlikowski R, Knapp P. Women's attitude towards prevention and rehabilitation of stress urinary incontinence. Progress Health Sci. 2014;1:130-4.

Cite this article as: Sundaramurthy $\mathrm{R}$, Kathiresan S, Venkateshvaran S, Kannan S. Knowledge, attitude and practice of post-natal exercises among post-natal women from a tertiary care centre, South India. Int J Reprod Contracept Obstet Gynecol 2020;9:1366-71. 